\title{
MATH5 controls the acquisition of multiple retinal cell fates
}

Liang Feng ${ }^{1,2}$, Zheng-hua Xie ${ }^{1,2}$, Qian Ding ${ }^{1,2}$, Xiaoling Xie ${ }^{1,2}$, Richard T Libby ${ }^{1,2}$, Lin Gan ${ }^{1,2,3^{*}}$

\begin{abstract}
Math5-null mutation results in the loss of retinal ganglion cells (RGCs) and in a concurrent increase of amacrine and cone cells. However, it remains unclear whether there is a cell fate switch of Math5-lineage cells in the absence of Math5 and whether MATH5 cell-autonomously regulates the differentiation of the above retinal neurons. Here, we performed a lineage analysis of Math5-expressing cells in developing mouse retinas using a conditional GFP reporter (Z/EG) activated by a Math5-Cre knock-in allele. We show that during normal retinogenesis, Math5-lineage cells mostly develop into RGCs, horizontal cells, cone photoreceptors, rod photoreceptors, and amacrine cells. Interestingly, amacrine cells of Math5-lineage cells are predominately of GABAergic, cholinergic, and A2 subtypes, indicating that Math5 plays a role in amacrine subtype specification. In the absence of Math5, more Math5-lineage cells undergo cell fate conversion from RGCs to the above retinal cell subtypes, and occasionally to cone-bipolar cells and Müller cells. This change in cell fate choices is accompanied by an up-regulation of NEUROD1, RXRY and BHLHB5, the transcription factors essential for the differentiation of retinal cells other than RGCs. Additionally, loss of Math5 causes the failure of early progenitors to exit cell cycle and leads to a significant increase of Math5-lineage cells remaining in cell cycle. Collectively, these data suggest that Math5 regulates the generation of multiple retinal cell types via different mechanisms during retinogenesis.
\end{abstract}

\section{Introduction}

In the developing central nervous system (CNS), different types of neurons are generated from a common pool progenitors in a phylogenetically conserved order. Though it is thought that the sequential generation of CNS neurons is regulated by both extrinsic and intrinsic factors, it is not well understood what the intrinsic factors are and how they determine the neuronal birth order. Vertebrate retinas consist of six major neuronal cell types and one glial cell type that originate from a common pool of retinal progenitors $[1,2]$ and are arranged in three well-defined cellular layers. The primary light-sensing neurons, cones and rods, are located in the outer nuclear layer (ONL). The interneurons, amacrine, bipolar and horizontal cells, and Müller cells compose the inner nuclear layer (INL). The ganglion cell layer (GCL) contains displaced amacrine cells and retinal ganglion cells (RGCs). The generation of these retinal cells follows a defined sequence that ganglion,

\footnotetext{
*Correspondence: lin_gan@urmc.rochester.edu

${ }^{1}$ Flaum Eye Institute, University of Rochester School of Medicine and Dentistry, Rochester, NY 14642, USA

Full list of author information is available at the end of the article
}

horizontal, amacrine, and cone cells are the first-born retinal cell types, and rod, bipolar and Müller cells are generated later [3]. Loss-and gain-of-function studies have demonstrated that transcription factors of the basic helix-loop-helix (bHLH) and homeodomain (HD) classes play key roles in retinal cell fate determination. The retinogenic bHLH factors, such as MASH1, MATH3, NGN2, and NEUROD1, are essential for the specification of major retinal cell types via a combined function with HD proteins CHX10, SIX3 and PAX6 [4-8]. Though alterations in the expression of above genes often lead to an increase or decrease in one or more retinal cell types, it is not clear whether the change in cell types arise from cell fate switch due to the lack of cell lineage analysis.

The vertebrate homolog of Drosophila atonal (ato), Ath5 (atonal homolog 5), is a key regulator of retinogenesis. Null mutations of ath 5 lead to agenesis of nearly all RGCs in mice and fish and to a concurrent increase of cone and amacrine cells [9-11]. Previous cell lineage studies using the Cre-loxP recombination system in mice showed that during normal retinal development Math5lineage cells differentiate into ganglion, horizontal, cone, 
and amacrine cells [12]. Nevertheless, it remains unknown what the cell fate choices of these Math5-lineage cells are in Math5-null retinas and how MATH5 regulates the differentiation of non-RGCs. Furthermore, the effect of Math5-null mutation on retinal progenitors is not fully understood. Here, we demonstrate that loss of Math5 leads to an increase of cone, rod, and the displaced amacrine cells originating from Math5-lineage cells and infrequently to the ectopic formation of conebipolar and Müller glial cells from Math5-lineage cells. The observed cell fate conversion is accompanied by the premature expression of non-RGC retinogenetic factors. Without Math5, an increased number of Math5-lineage cells remain in cell cycle or undergo apoptosis. The number of proliferating progenitors is transiently increased during early retinogenesis and is reduced in the postnatal retina. Thus, our analysis unveils a comprehensive function of Math5 in the generation of multiple retinal cell types during the retinal development.

\section{Results}

\section{Altered cellular composition in Math5-null retina}

To investigate the multiple effects of Math5-null mutation, we first assessed the change in different retinal cell types by co-labeling adult control and Math5-null retinal sections with cell type-specific markers (Table 1) and quantified the labeled cells separately in three cellular layers. In the GCL of Math5-null retinas, there was an increase in BHLHB5+ (GABAergic subtype), PROX1 + (A2 subtype) and GAD65+ (GABAergic subtype) amacrine cells (6-, 2-and 3-fold, respectively; Figure 1A-C, I). The ISL1+ and calretinin+ (ganglion and cholinergic amacrine) cells were reduced by $\sim 50 \%$ (Figure 1D, E, I), likely due to the absence of RGCs. Consequently, the total number of PAX6+ cells in the GCL, including all amacrine and ganglion cells, was not significantly changed (Figure 1I and Additional file 1: Supplemental Figure S1A). In the INL, the amacrine cells of GABAergic (GAD65+) subtype were reduced by $29.5 \% \pm 9.2 \%$ and of cholinergic (calretinin+) subtype by $34.4 \% \pm 13.4 \%$ (Figure 1C, D, I).

In the INL of Math5-null retinas, the total number of amacrine (PAX6+) cells was reduced by $21.9 \% \pm 6.2 \%$ (Additional file 1: Supplemental Figure S1A) and specifically, a decrease in GAD65+ $(29.5 \% \pm 9.2 \%)$ and calretinin $+(34.4 \% \pm 13.4 \%$; ) subtypes (Figure $1 \mathrm{C}, \mathrm{D}, \mathrm{I})$. There was also an overall reduction in bipolar cell number $(36.7 \% \pm 7.5 \%$ less $\mathrm{CHX10+}$ cells; Additional file 1: Supplemental Figure S1B) and specifically, total ON-bipolar cells $(\mathrm{Go} \alpha+$ ) reduced by $37.1 \% \pm 7.0 \%$ (Figure $1 \mathrm{I}$ and Additional file 1: Supplemental Figure S1C); ON-rod bipolar cells $(\mathrm{PKC} \alpha+)$ by $43.5 \% \pm 11.0 \%$; OFF-cone bipolar cells (VSX1+) by $48.2 \% \pm 3.9 \%$; and recoverin+ Type 2 OFF-bipolar cells by $76.9 \% \pm 11.2 \%$ (Figure $1 \mathrm{I}$ and Additional file 1: Supplemental Figure S1C, S1D). The reduction of amacrine and bipolar cells in the INL of Math5-null retina was also revealed by immunolabeling with anti-BHLHB5 $(46.8 \% \pm 9.0 \%$ reduction $)$, antiPROX1 (21.3\% $\pm 3.8 \%$ reduction), and anti-ISL1 $(19.7 \%$ $\pm 5.4 \%$ reduction) (Figure $1 \mathrm{~A}, \mathrm{~B}, \mathrm{E}, \mathrm{I})$. Interestingly, while all other neuronal cell types in the INL were reduced, the number of horizontal cells (calbinidin+) remained the same in control and Math5-null retinas (Figure 1I and Additional file 1: Supplemental Figure $\mathrm{S} 1 \mathrm{~F})$. In the ONL, rhodopsin+ and NRL+ rod photoreceptors were reduced by $30.1 \% \pm 5.2 \%$ (Figure $1 \mathrm{G}, 1 \mathrm{I}$, and Additional file 1: Supplemental Figure S1G) whereas $\mathrm{RXR} \gamma+$ cone photoreceptors were increased by 2-fold in Math5-null retinas (Figure 1H, I). Thus, targeted disruption of Math5 resulted in significant changes in almost every type of retinal neurons.

\section{Fate conversion of Math5-lineage cells in Math5-null retinas}

To investigate whether there is a fate conversion of Math5-lineage cells in Math5-null retinas, we used Math5-Cre knock-in mice and Z/EG GFP reporter mice to trace the fate of Math5-lineage cells. Upon activation, $Z / E G$ mice express a conditional GFP reporter gene uniformly in all retinal cell types (Additional file 2: Supplemental Figure S2). First, we compared the distribution of Math5-lineage cells in the control (Math5 $\left.{ }^{\mathrm{Cre} /+} ; \mathrm{Z} / \mathrm{EG}\right)$ and Math5-null (Math5 ${ }^{\text {Cre/lacZ }}$; Z/EG) retinas. Anti-GFP labeling showed similar onset of GFP expression in control and Math5-null retinas at E12 (Figure 2A), one day after the onset of endogenous Math5 expression. From E12.5 to E13.5, while GFP was expressed in the neuroblast layer (NBL), the presumptive GCL, and newly formed RGC axons in control retinas, GFP expression in Math5-null retinas were observed in the NBL but not in the GCL nor RGC axons (Figure 2B-D), suggesting the agenesis of RGCs. From E15.5 to E17.5, compared with GFP + cells seen in the GCL and the presumable cones in the outermost NBL of control retinas, the number of GFP+ cells in Math5-null retinas was increased in the $\mathrm{NBL}$ and in the outermost NBL but was reduced in the innermost NBL (Figure 2E, F). At P0, GFP+ cells with the morphology resembling horizontal cells could be identified in the INL of the control and Math5-null retinas (Figure 2G; arrows). At P7, Math5-null retina had more GFP+ cells in the ONL and some GFP+ bipolarlike cells in the INL (Figure $2 \mathrm{H}$; arrow). At P28, GFP+ cells resembling the morphologies of photoreceptors, horizontal, amacrine and ganglion cells were seen in control retinas (Figure 2I). In Math5-null retinas, no discernible change in the number of GFP+ cells was observed in the GCL and INL compared to control retina, however, GFP+ cells in the ONL were increased 
Table 1 List of antibodies used in this study

\begin{tabular}{|c|c|c|c|c|}
\hline Antibodies & Sources & $\begin{array}{l}\text { Working } \\
\text { dilution }\end{array}$ & Retinal cell markers & References \\
\hline anti-BHLHB5 & Santa Cruz Biotech. (Santa Cruz, CA) & $1: 1,000$ & $\begin{array}{l}\text { displaced amacrine, GABAergic amacrine, and OFF- } \\
\text { cone bipolar cells }\end{array}$ & {$[16]$} \\
\hline anti-BrdU & DSHB (Univ. of lowa) & $1: 50$ & cell cycle, S-phase cells & [16] \\
\hline anti-BRN3A & Chemicon Intl. (Temecula, CA) & $1: 400$ & retinal glanglion cells & {$[32]$} \\
\hline anti-BRN3B & Santa Cruz Biotech. (Santa Cruz, CA) & $1: 2,000$ & retinal ganglion cells & [32] \\
\hline anti-calbindin $28 \mathrm{~K}$ & Sigma (St. Louis, MO) & $1: 5,000$ & horizontal cells, some amacrine cells & [33] \\
\hline anti-calretinin & Calbiochem (San Diego, CA) & $1: 2,000$ & $\begin{array}{l}\text { ganglion and amacrine cells of cholinergic, non-All and } \\
\text { displaced subtypes }\end{array}$ & {$[33-35]$} \\
\hline $\begin{array}{l}\text { anti-activated } \\
\text { caspase-3 }\end{array}$ & R\&D Systems (Minneapolis, MN) & $1: 200$ & apoptotic cells & [36] \\
\hline anti-CHX10 & Exalpha (Watertown, MA) & $1: 200$ & progenitors, bipolar cells & {$[37]$} \\
\hline anti-GAD65 & BD Biosciences (San Jose, CA) & $1: 200$ & GABAergic amacrine cells & {$[38]$} \\
\hline anti-Go $\alpha$ & Upstate (Lake Placid, NY) & $1: 200$ & ON-bipolar cells & [33] \\
\hline anti-GFP & $\begin{array}{l}\text { MBL (Woaburn, MA) or Abcam, } \\
\text { (Cambridge, MA) }\end{array}$ & $1: 1,000$ & green fluoresence protein & [36] \\
\hline anti-lacz & $\begin{array}{l}\text { DSHB, Univ. of lowa or Chemicon Intl. } \\
\text { (Temecula, CA) }\end{array}$ & $1: 500$ & B-galactosidase & [36] \\
\hline anti-ISL1/2 & DSHB (Univ. of lowa) & $1: 400$ & $\begin{array}{l}\text { retinal ganglion, ON-bipolar, and cholinergic amacrine } \\
\text { cells }\end{array}$ & [39-41] \\
\hline Anti-Ki67 & BD Pharmingen (San Jose, CA) & $1: 200$ & cell cycle, all phases & [42] \\
\hline anti-NEUROD1 & Santa Cruz Biotech. (Santa Cruz, CA) & $1: 500$ & cone and amacrine cells & [15] \\
\hline anti-NRL & Chemicon Intl. (Temecula, CA) & $1: 1,000$ & rod photoreceptor cells & [43] \\
\hline anti-p27kip1 & BD Biosciences (San Jose, CA) & $1: 100$ & Muller cells & [44] \\
\hline $\begin{array}{l}\text { anti-phosphorylated } \\
\text { histone } 3\end{array}$ & Santa Cruz Biotech. (Santa Cruz, CA) & $1: 400$ & cell cycle, M-phase & [16] \\
\hline anti-PAX6 & DSHB (Univ. of lowa) & $1: 200$ & progenitors, pan-ganglion, and pan-amacrince cells & {$[16,45-47]$} \\
\hline anti-PKC $\alpha$ & Sigma (St. Louis, MO) & $1: 5,000$ & rod bipolar cells & [33] \\
\hline anti-PROX1 & Covance (Berkeley, CA) & $1: 1,000$ & $\begin{array}{l}\text { progenitors, bipolar, horizintal, All and displaced } \\
\text { amacrince cells }\end{array}$ & [48] \\
\hline anti-recoverin & Chemicon Intl. (Temecula, CA) & $1: 200$ & Type 2 OFF-cone bipolar, photoreceptor cells & {$[33,49]$} \\
\hline anti-rhodopsin & Chemicon Intl. (Temecula, CA) & $1: 200$ & Rod photoreceptor cells & {$[50]$} \\
\hline anti-RXRy & Santa Cruz Biotech. (Santa Cruz, CA) & $1: 200$ & Cone photoreceptor cells & [51] \\
\hline anti-VSX1 & $\begin{array}{l}\text { Gift from R.L. Chow (Univ. of Victoria, } \\
\text { Canada) }\end{array}$ & 1:100 & OFF-cone bipolar cells & {$[52,53]$} \\
\hline
\end{tabular}

by 2 -fold and bipolar-like GFP+ cells (arrow) were observed in the INL (Figure 2I, J).

To determine the identities of Math5-lineage cells in the ONL, we immunolabeled retinas with anti-RXR $\gamma$ and anti-rhodopsin for cone and rod cells, respectively. In Math5-null retinas, the cones and rods of Math5-lineage (GFP+) were increased by 1.5-and 4-fold, respectively (Figure 3A-D, 4F, and Additional file 3: Supplemental Figure S3A). Within the INL, anti-PAX6 labeling did not detect any overt change in the total number of Math5-lineage amacrine cells in the absence of Math5 (Additional file 3: Supplemental Figure S3B). However, Math5-lineage GABAergic amacrine cells (BHLHB5+ or GAD65+) were significantly reduced in Math5 mutants (Figure 3E-H, 4F). In contrast, there was an increase of Math5-lineage A2 (PROX1+), cholinergic (ISL1+) and calretinin+ amacrine cell subtypes in Math5-null compared to control (Figures 3I, 3J, 4F and Additional file 3: Supplemental Figure S3 D and S3C). In the GCL of Math5-null retinas, there was an increase in displaced amacrine cells (BHLHB5+ and GAD65+) of Math5-lineage (Figure 3E-H, 4F). In addition, anti-PROX1 labeled GFP+ displaced amacrine cells were found in the GCL of Math5-null retinas, which were not detected in control retinas (Figure 4F and Additional file 3: Supplemental Figure S3C).

In control retinas, Math5-lineage cells were not observed in bipolar cells but loss of Math5 led to the appearance of bipolar cells expressing CHX10 and GFP (Figure 4A). Interestingly, these cells expressed Goo, Vsx 1 , or recoverin (Figure 4B-D) but not PKC $\alpha$ (Figure 4E), demonstrating that a small percentage of Math5-lineage cells switch their fate to cone-bipolar but not to rod-bipolar cells in the absence of Math5. Moreover, compared to the absence of 

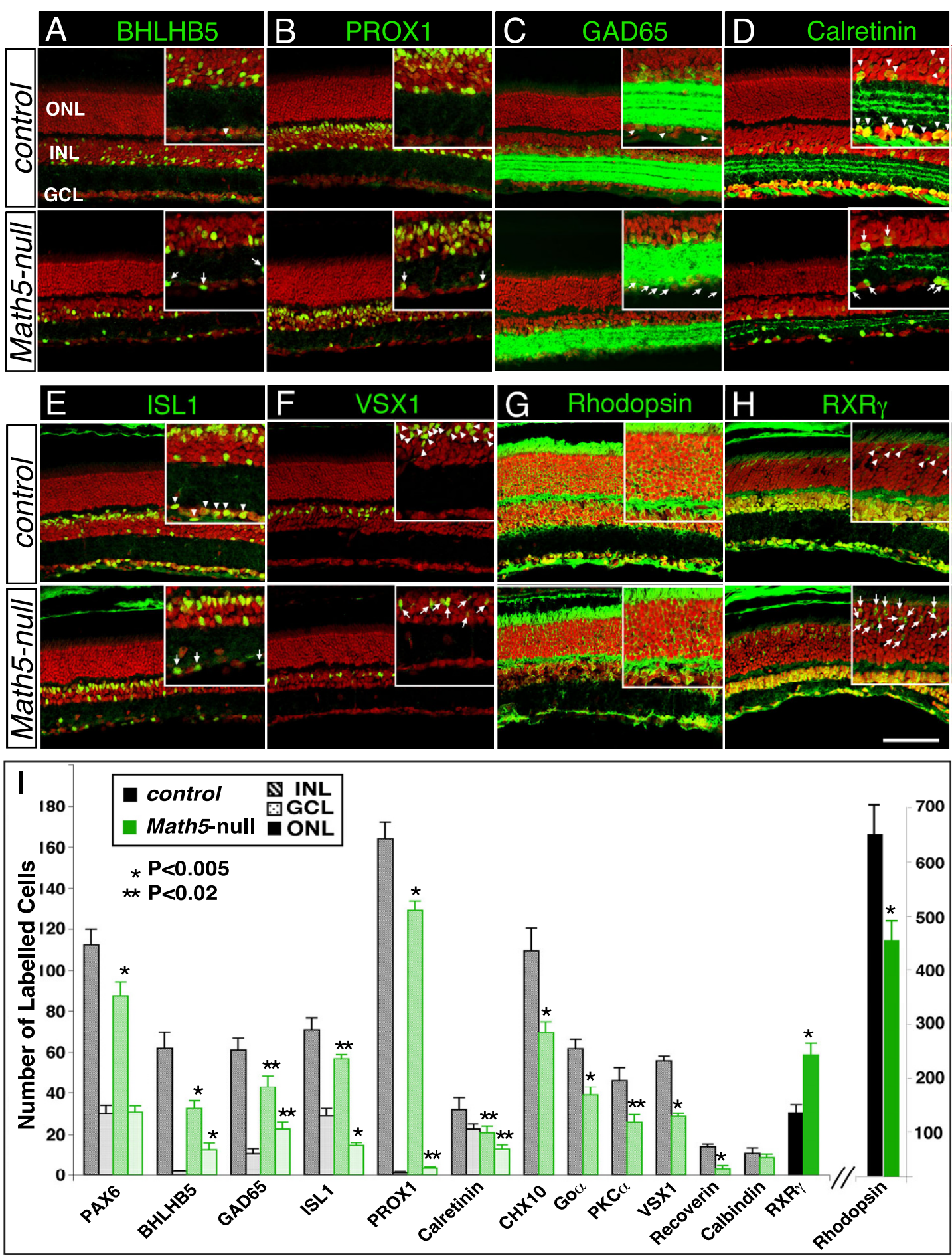

Figure 1 Effects of Math5-null mutation on retinal cell types. (A-H) Retinal sections from P28 were immunolabeled with cell type-specific markers (green) and nuclear counterstained with propidium iodide ( $\mathrm{PI}$, red). Loss of Math5 leads to an increase in the displaced amacrine cells immunoreactive for BHLHB5 (A), PROX1 (B) and GAD65 (C) in the GCL, and to a severe loss of calretinin+ (D) and ISL1+ (E) cells in the INL and the GCL. Furthermore, in the ONL, there is a marked reduction in VSX1+ cone bipolar cells (F) and rhodopsin ${ }^{+}$rod photoreceptors (G), whereas the RXR $\gamma+$ cone photoreceptors are increased (H). (I) Quantification of cells expressing specific markers in the ONL, INL and the GCL per $350 \mu \mathrm{m}$ length of retinal sections. Eyes from five mice were analyzed per genotype. Arrows in the insets indicate cells expressing specific markers in the wild type retina, while arrowheads in the insets indicate those of Math5-null retina. INL, inner nuclear layer. GCL, ganglion cell layer. ONL, outer nuclear layer. Scale bar equals $100 \mu \mathrm{m}$. 


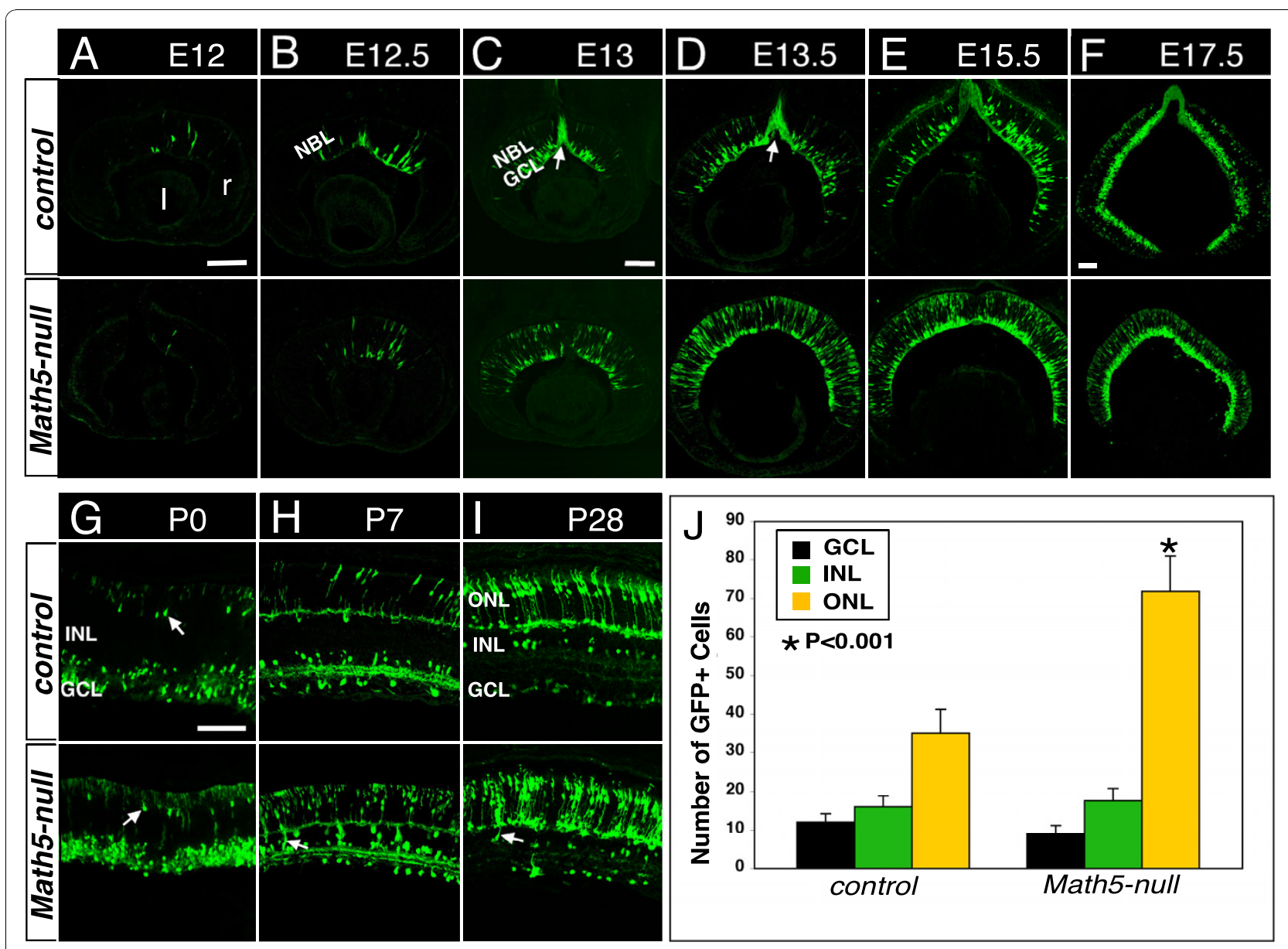

Figure 2 The spatiotemporal pattern of Math5-lineage cells in the developing control and Math5-null retinas. Retinal sections from the control (top panels) and Math5-null (bottom panels) retinas at indicated developmental stages were immunolabeled with anti-GFP (green) to mark the Math5-lineage cells. (A) The onset of GFP expression starts at E12 in the NBL of the control and Math5-null retinas. (B and C) At E12.5E13, GFP+ cells are present in the NBL, the newly formed GCL, and the RGC axon bundles (arrow) of the control retina. In the Math5-null retina no GCL neurons or RGC axon bundles are observed and there are significantly more GFP+ cells in the outermost NBL. (D-F) At E13.5-E17.5, compared to GFP+ cells located in the GCL and the outermost NBL of the control retina, GFP+ cells in the Math5-null retina are detected throughout the retina but no visible GFP+ RGC axon bundle are present. Moreover, there is an increase in GFP+ cells in the NBL and a decrease in the GCL. (G) At P0, GFP is expressed in the horizontal-like cells (arrows) in the INL of control and Math5-null retinas. (H and I) At P7 and P28, GFP+ cells are located in three retinal layers. Compared to the control, twice as many GFP+ cells were seen in the ONL in Math5-null retina and a few GFP+ bipolar-like cells (arrow) are present in the INL. (J) Quantification of GFP+ cells in the ONL, INL and the GCL per $350 \mu \mathrm{m}$ length of adult retinal sections. I, lens. r, retina. NBL, neuroblast layer. Scale bars equal $100 \mu \mathrm{m}$ in A (applies to A, B), C (applies to C, D, E), F (applies to F), and $\mathrm{G}$ (applies to $\mathrm{G}-1)$.

Müller cells originating from Math5-lineage in the control, loss of Math 5 also resulted in a very small number of Math5-lineage cells adopting the fate of Müller cells (p27kip1+/GFP+; Additional file 3: Supplemental Figure S3F). Overall, we observed no change in the total number of horizontal cells (Figure 4F and Additional file 3: Supplemental Figure S3E).

\section{Changes in cell proliferation and apoptosis in Math5-null retinas}

Due to the lack of working anti-MATH5 antibody, it remains unknown whether MATH5 is expressed in progenitors or postmitotic cells. We genetically tagged
MATH5 by fusing a HA-tag in-frame to MATH5's Cterminus using the knock-in approach in mice and used anti-HA to monitor MATH5 expression. The homozygous Math $5^{\text {HA/HA }}$ mice were normal and had retinas indistinguishable from their wild type controls (data not shown), indicating that the HA-tag does not interfere with the function of MATH5. Co-immunolabeling of Math5-HA retinas at E12.5 with anti-HA and cell cycle markers demonstrated MATH5's expression in the NBL where it partially overlapped with BrdU and Ki67 (Figure 5A,B), indicating that some MATH5+ cells are proliferating progenitors. When the GCL is clearly identifiable at E13.5, labeling of Math5$H A$ retinas showed that MATH5 expression was mostly 


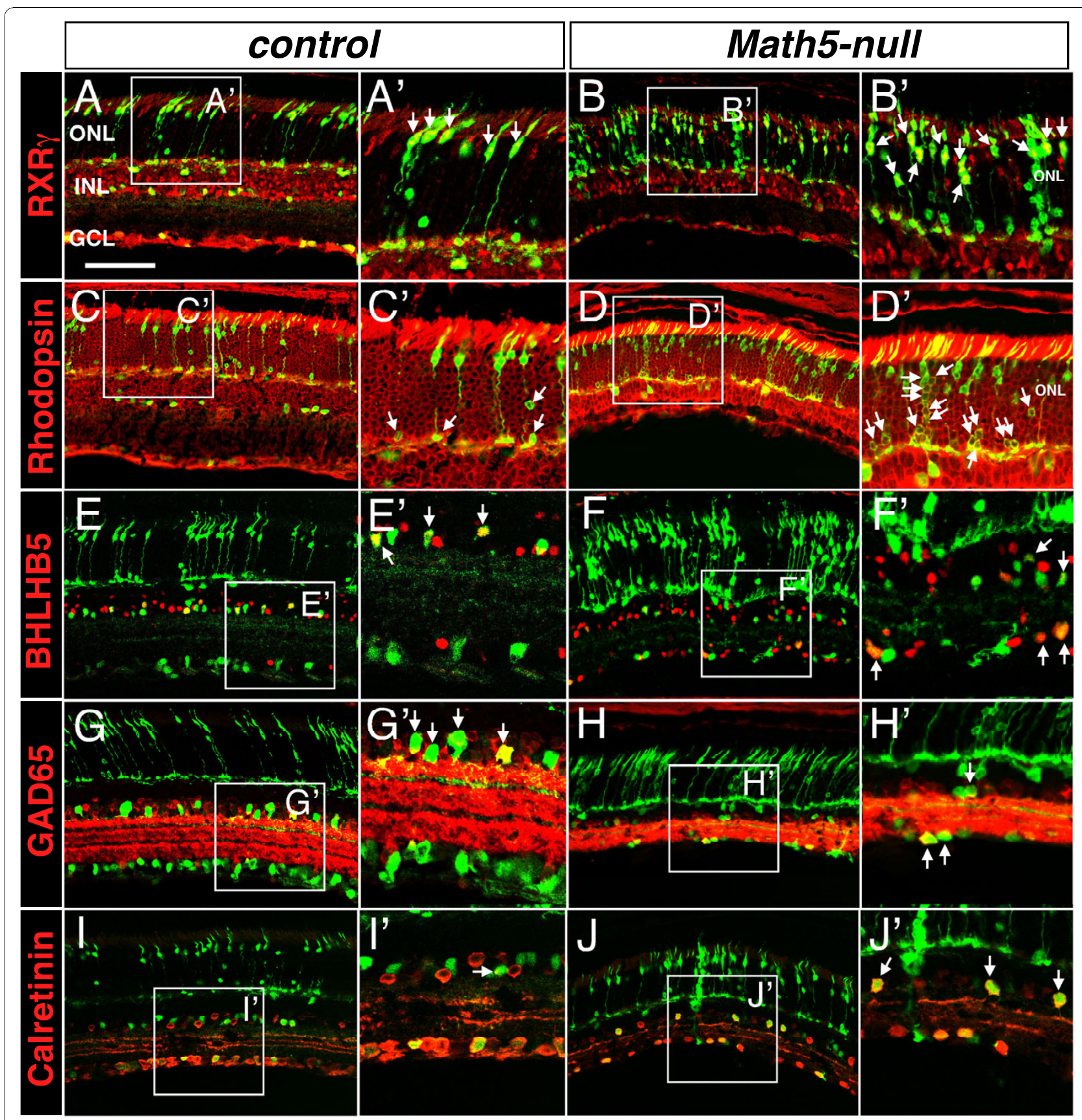

Figure 3 Fate conversion of Math5-lineage cells in Math5-null retinas. Adult retinal sections from the control and Math5-null mice were immunolabeled with cell type-specific markers (red) and anti-GFP (green). (A-D) Math5-lineage cone photoreceptors marked by RXRy (arrows in $A$ and $B$ ) are increased in number but the rod photoreceptors labeled by rhodopsin (arrows in $C$ and $D$ ) are reduced in number in Math5-null retinas. $(E-H)$ Among the Math5-lineage cells, BHLHB5+ $(E, F)$ and GAD65+ $(G, H)$ GABAergic amacrine cells (arrows) are reduced in number in the INL but are increased in the GCL of Math5-null retinas. $(I, J)$ Calretinin+ amacrine subtypes (arrows) are increased in number in the INL of Math5-null retinas. Enlarged views of boxed regions are shown in A' to J'. Scale bar equals $100 \mu \mathrm{m}$.

detected in the NBL where it partially colocalized with BRN3B, but was absent in the newly formed GCL where BRN3B and p27kip1 were highly expressed (Figure 5C, D). Thus, our data indicate that MATH5 is expressed in retinal progenitors as well as in the nascent, migrating RGCs but its expression is turned off in the post-migration RGCs in the GCL of the early retina.

A recent study has shown that MATH5 promotes cell cycle exit of retinal progenitors in E12.5 mouse retinal explants [13]. To test whether Math5-null mutation 


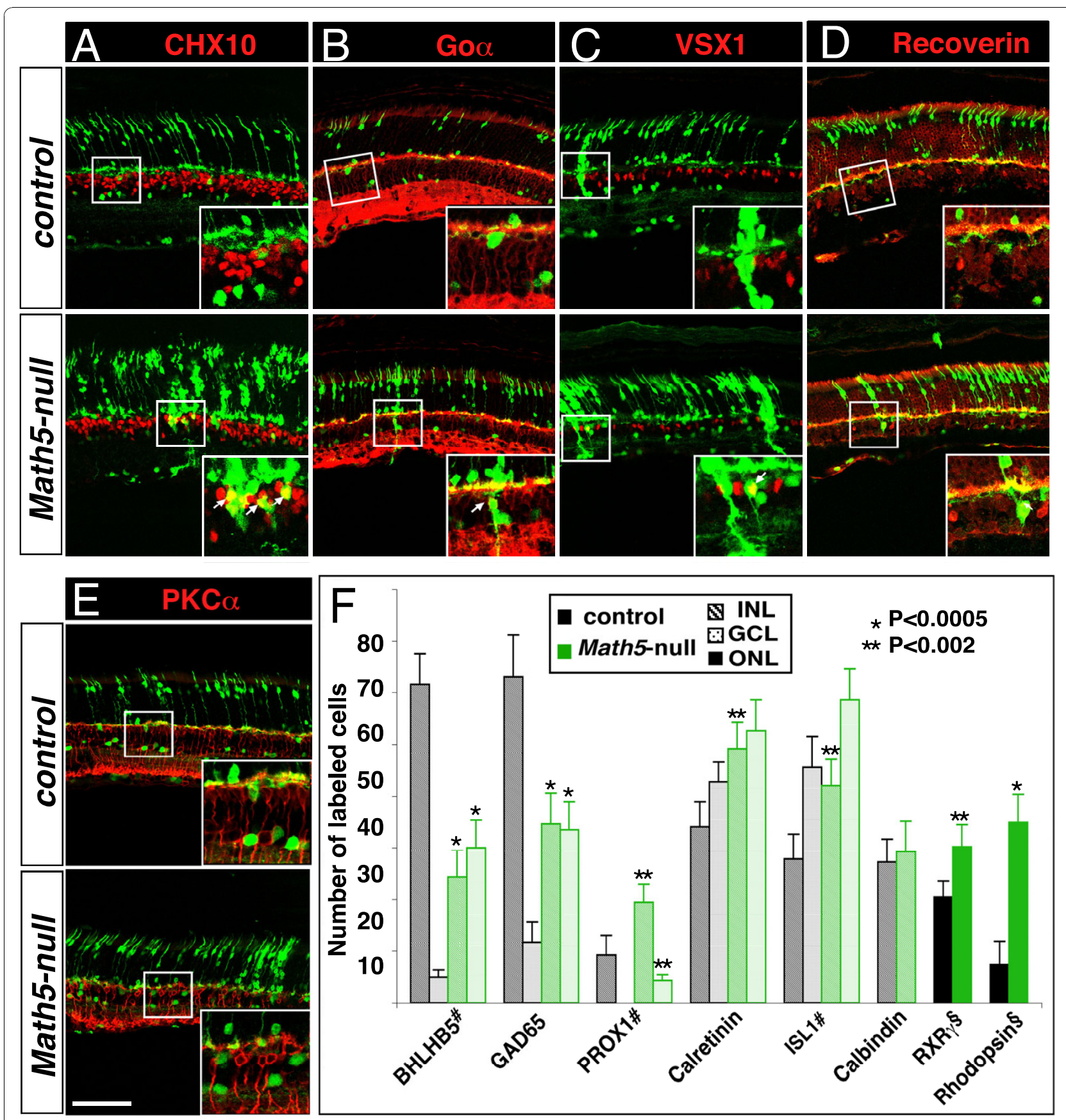

Figure 4 The cone-bipolar fate of Math5-lineage cells in the absence of Math5. Adult retinal sections from the control (top panels) and Math5-null (bottom panels) mice were immunolabeled with cell type-specific markers (red) and anti-GFP (green). (A-E) Compared to the control retina in which GFP expression is not co-localized with bipolar cell markers, a few of GFP+ cells in the Math5-null retina are positive for CHX10 (arrows), Go $\alpha$ (arrow), VSX1 (arrow) or recoverin (arrow) but not for PKC $\alpha$ (E). (K) Quantification of GFP+ cells expressing specific markers in the ONL, INL and the GCL per retinal section. Scale bar equals $100 \mu \mathrm{m}$. Notes: \#For BHLHB5+, PROX1+ and ISL1+ cells in the INL, only the amacrine cells were counted per retinal section; §RXR $\gamma+$ and rhodopsin+ cells were counted per $350 \mu \mathrm{m}$ linear length.

affects the cycle exit of Math5-lineage cells, we pulselabeled the developing control and Math5-null retinas with BrdU. While anti-BrdU labeling showed a $23.8 \% \pm$ 9.6\% increase of total mitotic cells at S-phase in Math5null retinas at E13.5 and $23.2 \% \pm 6.2 \%$ at E15.5, the
BrdU+ cells of Math5-lineage (GFP+) were increased by 40-fold at E13.5 and 7.5-fold at E15.5 (Figure 6A, B, G, H). Similarly, anti-phosphorylated histone 3 (PH3) revealed a $28.4 \% \pm 6.3 \%$ increase in all mitotic cells at M-phase in Math5-null retina at E14.5 and GFP+ cells 


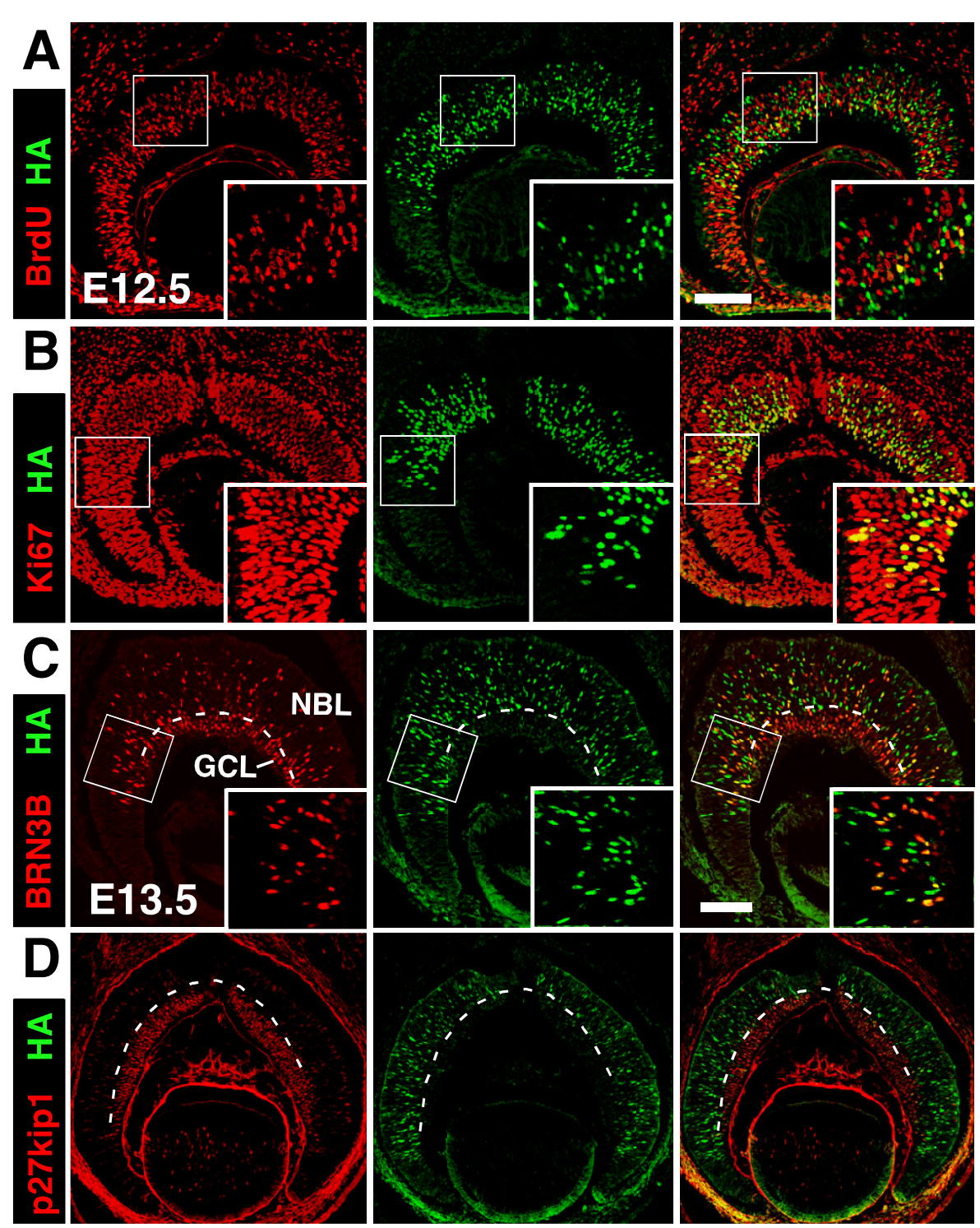

Figure 5 Expression of MATH5 in retinal progenitors and migrating RGCs. Retinal sections from the Math5-HA embryos at the indicated developmental stages were immunolabeled with anti-HA (green) to detect MATH5 and cell type-specific markers (red). (A and B) Partial colocalization of HA with BrdU (A) for mitotic cells at S-phase and Ki67 (B) for all mitotic cells reveals the expression of MATH5 is some progenitors at E12.5. (C and D) The expression of MATH5 is largely restricted in the NBL but not the newly formed GCL marked by BRN3B (C) and p27kip1 (D) expression at E13.5. Colabeling of HA and BRN3B shows the partial co-localization of MATH5 and BRN3B in the nascent, migrating RGCs in the NBL. Boxed regions are shown in the inserts. Dashed line is the arbitrary border between the GCL and the NBL. Scale bars are $100 \mu \mathrm{m}$.

at M-phase were increased by 10.5 -fold (Figure 6E-H). There was no significant change in the total number of proliferating cells at E12.5 and E17.5 (Figure 6G, 6H, and Additional file 4: Supplemental Figure S4). In postnatal retinas, loss of Math5 resulted in a reduction in the number of BrdU+ cells by $37.1 \% \pm 5.1 \%$ at $\mathrm{P} 0$ and $55.1 \% \pm 6.4 \%$ at P3 (Figure 6C, D, G, H).

Additionally, we examined the change in apoptosis of Math5-lineage cells by co-immunolabeling with antiGFP and anti-activated caspase-3. Compared to a low number of apoptotic cells of Math5-lineage in control retinas, loss of Math5 resulted in a significant increase in apoptosis of Math5-lineage cells at E12.5 to E17.5 (Figure 7) but no overt change was found after P0 (data not shown). Moreover, a majority of the apoptotic Math5-lineage cells were located in the innermost layer of developing retinas, the presumable GCL. Thus, the change in proliferation and apoptosis could contribute to the broad effect of Math5-null mutation on multiple retinal cell types. 


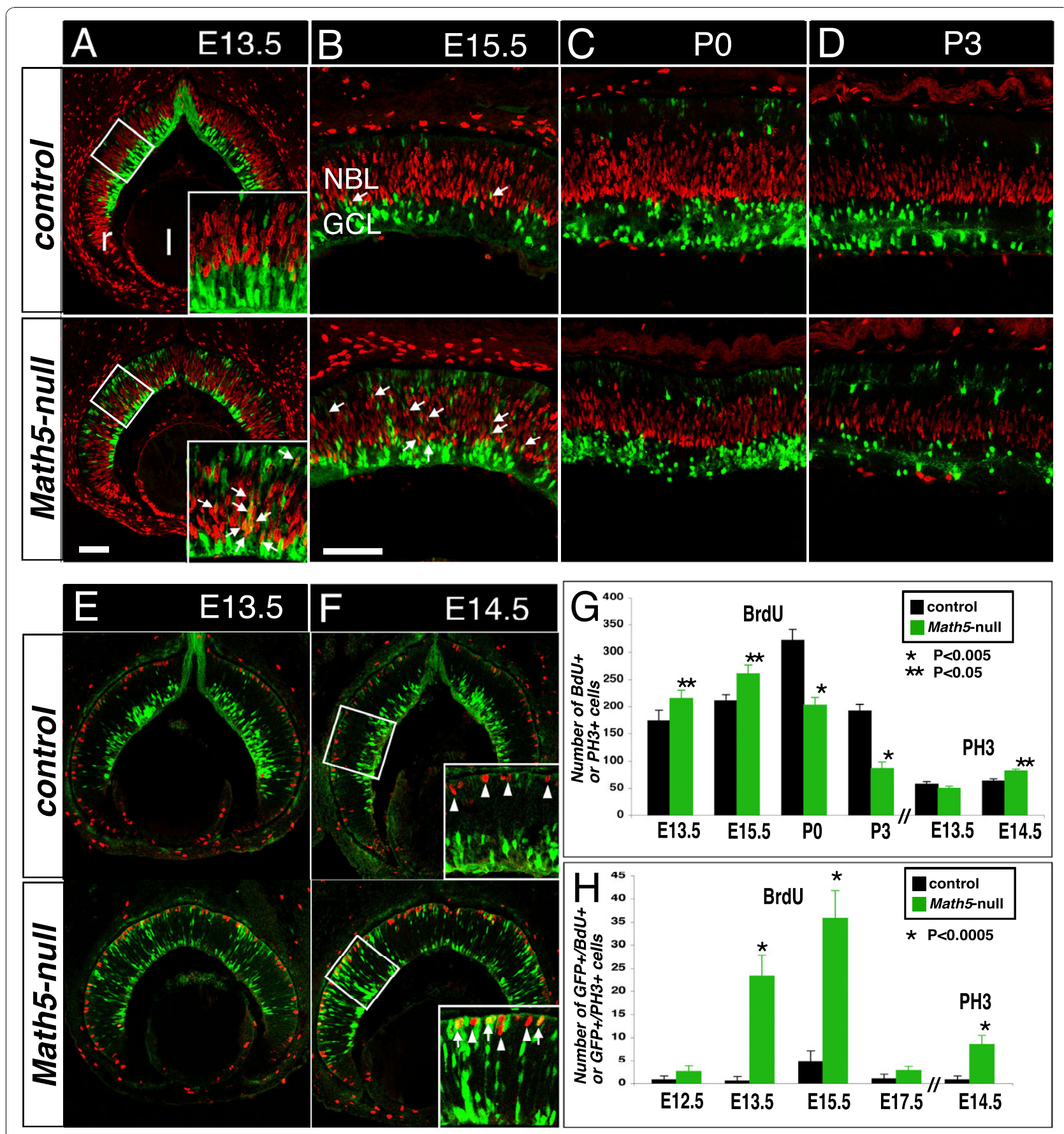

Figure 6 Loss of Math5 alters the proliferation of retinal progenitors and causes the increased Math5-lineage cells in cell cycle. Retinal sections from the control (top panels) and Math5-null (bottom panels) mice at the indicated developmental stages were immunolabeled with cell proliferation markers (red) BrdU (S-phase) or PH3 (M-phase) and anti-GFP (green). (A, B) At E13.5 to E15.5, GFP+/BrdU+ cells (arrows) are rarely detected in control retinas but are frequently seen in Math5-null retinas. (C, D) At P0 and P3, no visible GFP+/BrdU+ cells are found in the control and Math5-null retina, and the BrdU+ cells are profoundly decreased in Math5-null retinas. (E and F) PH3+ cells (red; arrowheads) are rarely seen as GFP+ cells in the control retina but in Math5-null retina, the GFP+/PH3+ cohorts (arrows) are significantly increased. (G) Quantification of the total BrdU+ or PH3+ cells per $350 \mu$ m length. (H) Quantification of the GFP+/BrdU+ or GFP+/PH3+ cells per retinal section. Scale bar equals $100 \mu \mathrm{m}$ in A (applies to A, E and F), B (applies to B-D). 


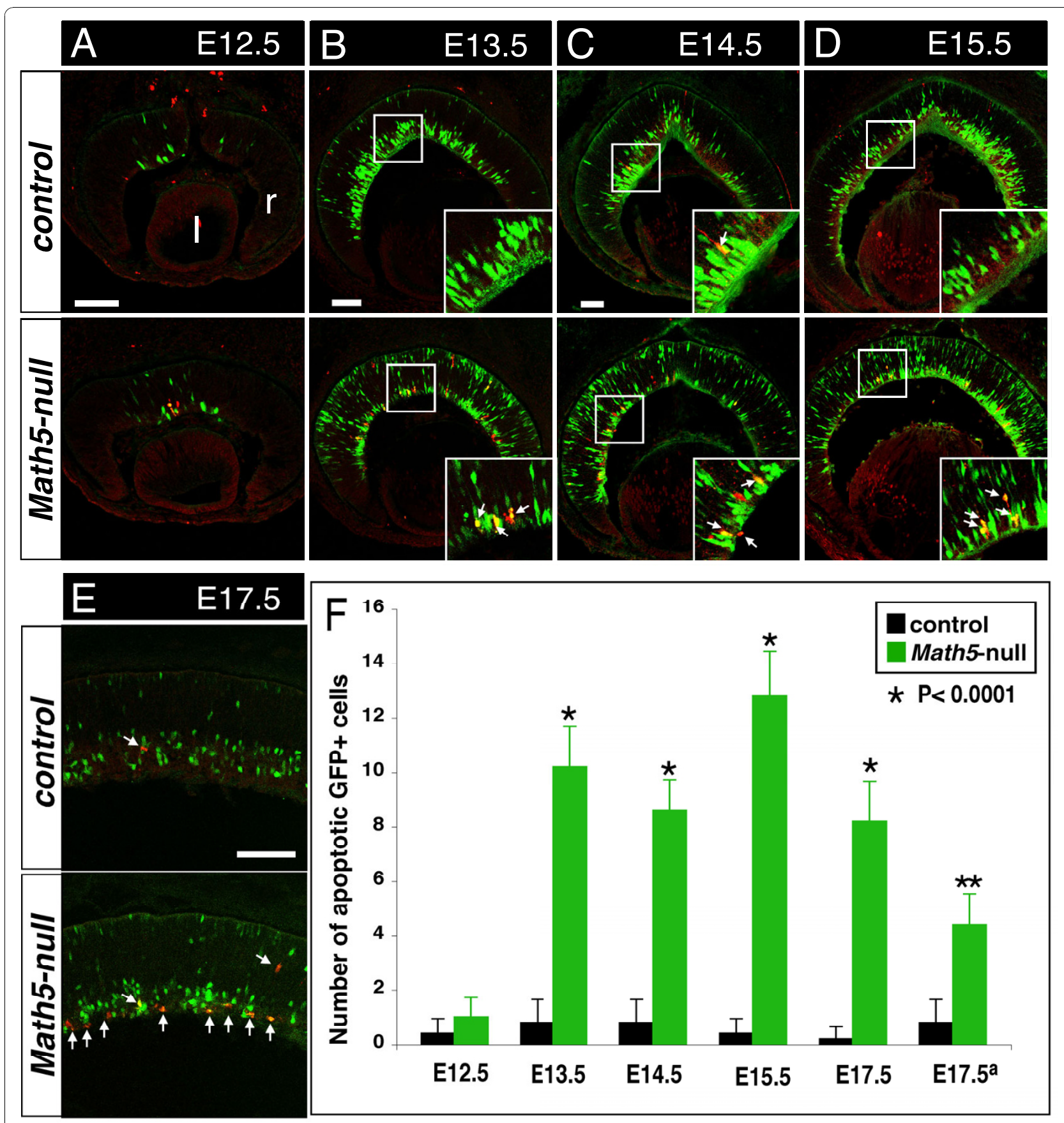

Figure 7 Math5-lineage cells undergo apoptosis in Math5-null retinas. Retinal sections from the control (top panels) and Math5-null (bottom panels) embryos at the indicated developmental stages were immunolabeled with anti-activated caspase-3 (red) and anti-GFP (green). (A-E) At E12.5 to E17.5, compared to the GFP+ apoptotic cells (arrows) occasionally seen in control retinas, the number of GFP+ apoptotic cells is significantly increased in Math5-null retinas. Insets in B-D indicate the enlarged views of boxed regions. Scale bar equals $100 \mu \mathrm{m}$. (F) Quantification of the GFP+/caspase-3+ cells per retinal section. Note: $a$, apoptotic cells negative for GFP were counted.

Premature expression of non-RGC regulator proteins in Math5-null retinas

To investigate the molecular mechanisms underlying the increased number of displaced amacrine cells in Math5null retina, we analyzed the expression of NEUROD1 and BHLHB5, two essential molecules in the genesis of photoreceptor and amacrine cells [14-16]. Compared to the expression of BHLHB5 and NEUROD1 in a very few Math5-lineage cells at E13.5 in control retinas, there was a significant increase in Math5-lineage cells expressing BHLHB5 and NEUROD1 in Math5-null retinas (Figure 8A, B). Such an increase was first detected at 


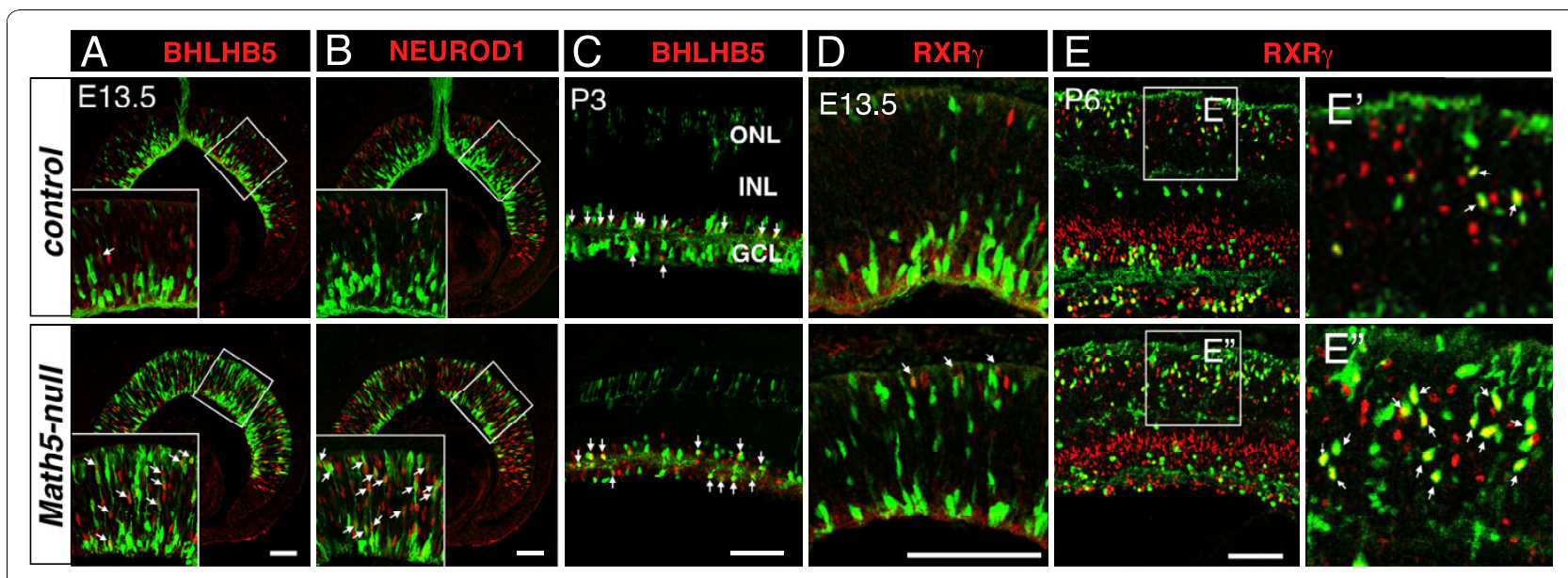

Figure 8 Loss of Math5 leads to an up-regulated expression of non-RGCs factors in Math5-lineage cells. Retinal sections from the control (top panels) and Math5-null (bottom panels) embryos at the indicated developmental stages were immunolabeled with cell type markers (red) and anti-GFP (green). (A, B) At E13.5, many GFP+ Math5-lineage cells express BHLHB5 (A) or NEUROD1 (B) (arrows) in the Math5-null retina but not in the control retina. (C) At P3, compared to the control, more GFP+/BHLHB5+ cells (arrows) are seen in the GCL and less in the INL of Math5-null retinas. (D, E) The number of GFP+/RXR + + cone cells (arrows) is increased in Math5-null retinas at E13.5 (D) and P6 (E). Enlarged view of boxed regions in $E$ is shown in $E^{\prime}$. Scale bars equal $100 \mu \mathrm{m}$.

E12.5, the earliest stage when Math5-lineage cells could be identified by the Cre-loxP approach (Additional file 5: Supplemental Figure S5A, B). At E17.5, anti-BHLHB5 labeling marks cells mostly located at the opposing borders of the GCL and the developing INL (Additional file 5: Supplemental Figure S5C), these are amacrine cells. In the absence of Math5, RGCs were rarely detected by anti-BRN3B labeling (Additional file 5: Supplemental Figure S5D), while more BHLHB5+ cells of Math5-lineage were seen in the GCL (Additional file 5: Supplemental Figure S5C). This change was more obvious at postnatal stages (Figure $8 \mathrm{C}$, arrows). Similarly, more amacrine cells were identified by anti-ISL1 labeling (Additional file 5: Supplemental Figure S5E), but not by anti-calretinin that marks the late-born amacrine cells (Additional file 5: Supplemental Figure S5F).

We also analyzed the expression of NEUROD1 and $\mathrm{RXR} \gamma$ that are required for the genesis of photoreceptors. At E17.5, many NEUROD1+ cells were located near the outermost NBL in both control and Math5null retinas. The location of these cells suggests that they are photoreceptor cells (Additional file 5: Supplemental Figure S5G). Co-labeling the retinas with anti-RXR $\gamma$ (cone marker) at E13.5, E17.5, and P6 demonstrated that many of the additional GFP+ photoreceptors are in fact cone photoreceptors of Math5lineage (Figure 8D, 8E, and Additional file 5: Supplemental Figure S5H).

\section{Discussion}

In this study, we have used the Cre/loxP approach to trace and compare the fates of Math5-lineage cells in normal and Math5-null retinas. The results presented here demonstrate that in the absence of Math5, there is an increase of Math5-lineage cells adopting amacrine, cone, and rod cell fates or choosing the new fates of cone bipolar and Müller cells. Furthermore, the loss of Math5 has a broad effect on retinal development including the development of multiple retinal cell types, cell proliferation, and cell survival.

Role of MATH5 in regulating multiple retinal cell fates Cell lineage tracing studies have revealed that all retinal neurons and Müller cells are derived from a common pool of progenitors, and the daughter cells of a progenitor often assume distinct neuronal identities [17-20]. These studies suggest that cell fate decisions are made during or after the terminal neurogenic cell cycle under the influence of environmental cues. Subsequent studies have shown that transcription factors play pivotal roles in the specification and differentiation of distinct retinal cell types [8,21-23]. Thus, the interplay between cellextrinsic cues and cell-intrinsic factors is critical for the outcome of retinal cell differentiation.

Previous analysis of Math5-lineage cells during normal retinal development reveals that Math5 is expressed in retinal progenitors giving rise to all horizontal and ganglion cells and to some amacrine and photoreceptor cells [12]. Nevertheless, it is not well understood whether the development of other retinal cells is affected and whether there is a fate conversion of Math5-lineage cells in the absence of Math5. Using lineage tracing analysis, we have found that in the INL of Math5-null retinas, there is a reduced number of GABAergic amacrine 
cells accompanied by an increase in cholinergic and A2 glycinergic amacrine cells from Math5-lineage (Figure 3). Similarly, in the GCL of Math5-null retinas, the loss of RGCs goes together with an increase of the displaced amacrine cells from Math5-lineage, and more rod and cone cells arise from Math5-lineage in the ONL of Math5-null retinas (Figures 3, 4 and Additional file 3: Supplemental Figure S3). All of these changes clearly demonstrate cell fate switch in Math5-lineage cells after the ablation of Math5. Moreover, we have observed a small number of Math5-lineage cells adopting the fate of cone bipolar cells and Müller cells in Math5-null retinas despite that these retinal cell types are generated postnatally during normal retinal development (Figure 4 and Additional file 3: Supplemental Figure S3).

The fate conversion of Math5-lineage cells from RGCs to other retinal neurons and Müller glial cells in Math5null mice demonstrated here, extends previous loss-offunction analysis of Math5 and suggests that MATH5 directly regulates the acquisition of multiple cell fates by retinal progenitors. MATH5 not only promotes RGC fate but also suppresses the fate choices of amacrine, cone, rod, cone bipolar, and Müller cells. We analyzed the expression of NEUROD1, RXR $\gamma$, and BHLHB5 that are implicated in the development of cones, pan-amacrine cells and the subset of GABAergic amacrine cells, respectively $[15,16]$. Interestingly, the fate conversion of Math5-lineage cells is associated with the precocious expression of these transcription factors (Figure 8). Since Math5-lineage cells give rise to RGC and other retinal neurons during normal development [12], MATH5 functions as a RGC competence factor and it alone is insufficient to specify RGC fate. We hypothesize that during normal retinogenesis, the transient MATH5 expression provides progenitors with a brief window of opportunity to choose RGC fate as the first choice (Figure 9). During this RGC competence period, MATH5 transiently suppresses the differentiation program of selected retinal neurons by directly or indirectly regulating Neurod1, Bhlhb5, and $R X R \gamma$ and possibly interacting with other non-RGC regulators such as Foxn4. Through this mechanism, MATH5 influences cell fate choice and determines birth order of retinal cells that RGCs are generated first. Likewise, the cell fate switch of Math5lineage cells from RGC to cone-bipolar cells could result from the up-regulation of BHLHB5 as BHLHB5 is associated with the genesis of a subset of off-cone bipolar cells [16].

Though cell birthdating experiments show that retinal neurons are generally produced in two phases: ganglion, horizontal, amacrine, and cone cells are generated during the early embryonic phase, and rod, bipolar and Müller cells are produced in the later postnatal phase [3]. It is not clear whether the early embryonic progenitors in the early environment are competent to differentiate into later born retinal cells. Surprisingly, we have observed that in Math5-null retinas, an increased number of Math5-lineage cells are converted into the late born rod, cone bipolar and Müller cells (Figure 4 and Additional file 3: Supplemental Figure S3). Previous birthdating studies in Math5-null retinas have also shown that some Müller cells are born precociously as early as at E11.5 [24]. Taking together, these findings suggest that the early retinal environment could permit the differentiation of late-born retinal cells but intrinsic factors, such as MATH5, suppress progenitors' potential to differentiate into these late born retinal cells. Future birthdating experiments of Math5-lineage cells in Math5-null retinas will confirm this possibility. Additionally, the cell fate conversion of Math5-lineage cells into cone bipolar cells in Math5-null retinas suggests that the differentiation of specific bipolar subtypes might require different intrinsic and or environment factors. Alternatively,

\section{The role of Math5 in cell cycle}

The multiple retinal cell fates of Math5-lineage cells imply that these Math5-expressing cells are not yet committed to a particular cell fate. We found that in the absence of Math5, the number of proliferating retinal progenitors is transiently increased at E13.5 to E15.5 but is reduced later in the first postnatal week (Figure 6). The postnatal drop in cell proliferation rate could be caused by the loss of RGCs in Math5-null retinas since SHH produced by the early born RGCs are required to maintain the proliferation of the late retinal progenitors and the loss of RGCs results in down-regulation of GLI1, a SHH effector, in retinal progenitors [25]. It is likely that loss of Math5 leads to the reduced expression of GLI1, which in turn, could account for the reduced proliferation later. The early transient increase in cell proliferation in Math5-null retinas is consistent with the previous report [24]. However, it remains uncertain whether MATH5 plays a role in driving the progenitors to exit cell cycle and whether Math5-lineage cells remain in or re-enter cell cycle without MATH5. Using lineage analysis, we have observed that in the absence of Math5, the number of mitotic Math5-lineage cells is significantly increased (Figure 6) at E13.5 to E15.5. In a recent study, the ectopic expression of Math5 and another neurogenic gene Ngn3 in E12.5 mouse retinal explants leads to a significant reduction in the number of progenitors in BrdUlabeled S-phase [13]. Thus, it is likely that a key role of MATH5 in retinal development is to drive the cell cycle exit of progenitors. Without MATH5, the retinal progenitors continue proliferating or delay the cell cycle exit. 


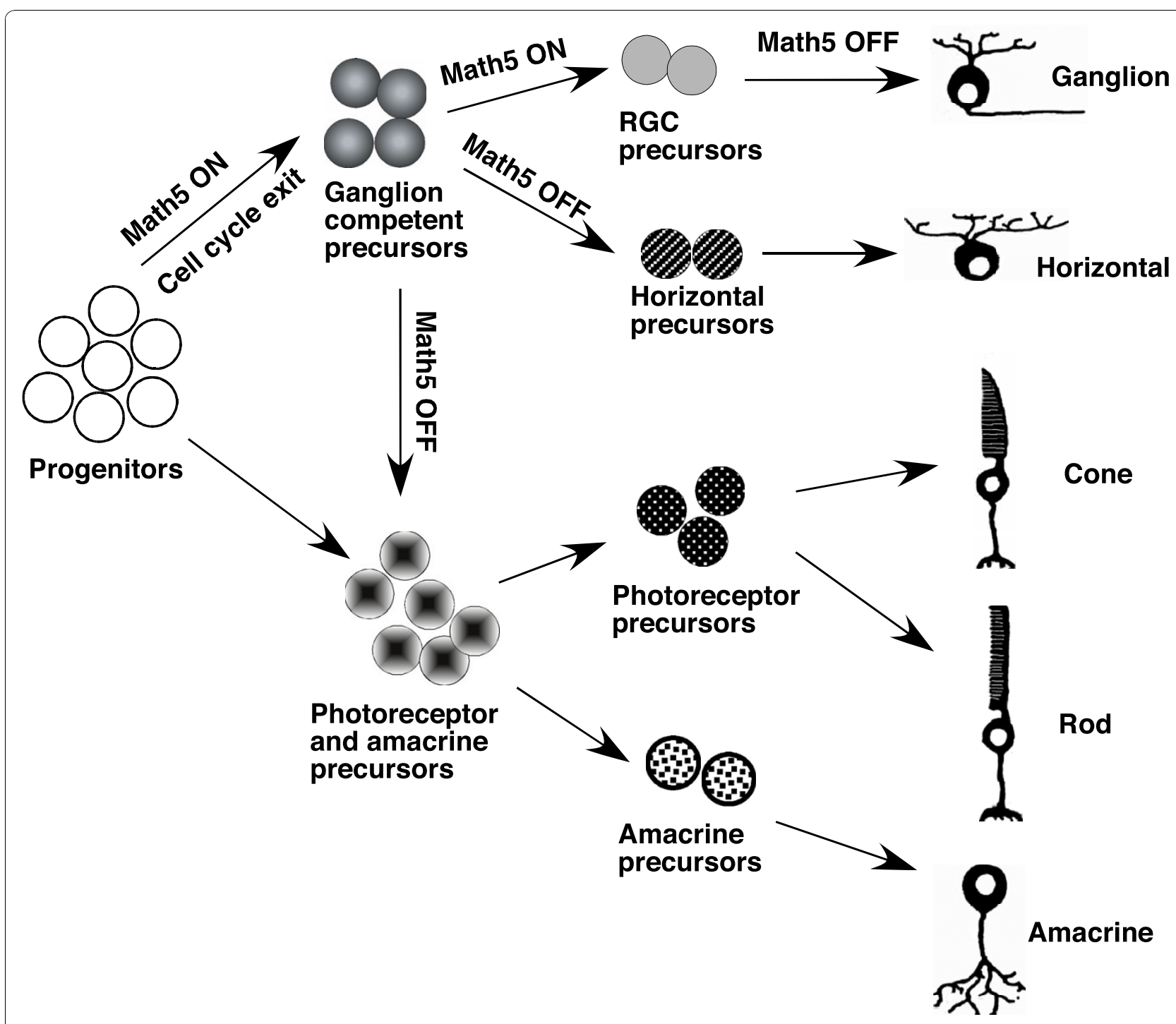

Figure $9 \mathrm{~A}$ model for the role of MATH5 in the sequential formation of early-born retinal neurons. During normal retinogenesis, the expression of MATH5 starts in selected retinal progenitors and promotes the cell cycle exit. The transient expression of MATH5 makes the postmitotic precursors become RGC-competent and temporarily blocks non-RGC differentiation programs by suppressing the expression of retinogenic factors such as NEUROD1, NGN2, MATH3, BHLHB5, and RXR $\gamma$. During this competence period, MATH5 activates a network of transcription factors including BRN3B and ISL1 in some precursors to initiate the RGC differentiation program. The remaining precursors lose RGC-competence when MATH5 expression ceases, express other retinogenic factors and generate horizontal cells (all horizontal cells are of Math5-lineage), or along with the non-Math5-lineage precursors, produce amacrine, rod, and cone cells.

Previous studies showed that Math5 mRNA expression is largely restricted in the NBL, suggesting its expression in progenitors $[12,26]$. However, studies using Math5-lacZ reporter mice revealed a largely postmitotic expression pattern of lacZ, implying Math5 expression after the cell cycle exit of progenitors [12,24]. These contradicting observations likely reflect the transient nature of Math5 mRNA and the long half-life of lac $Z$ reporter protein. To resolve this issue, we have genetically tagged endogenous MATH5 with HA-tag. Colocalization of HA-tag and cell cycle markers reveals significant more mitotic progenitors expressing MATH5 than previously reported using the Math5-lacZ reporter. One possible explanation of this apparent discrepancy is that lacZ protein is more stable than MATH5 and persists in postmitotic, differentiated cells, such as the newly formed RGCs [12,24]. On the other hand, MATH5 expression, revealed by the HA-tag, is likely highly transient and mirrors the dynamic mRNA expression pattern in cells of the NBL but not in the GCL $[12,26]$. The observation of Math5-lacZ expression mostly in postmitotic RGCs of the GCL and of 
MATH5-HA in cells of the NBL further demonstrates that the onset of Math5 expression begins in progenitors in the NBL and that MATH5 expression drives progenitors to exit cell cycle and to differentiate into RGCs and other retinal neurons.

Our results have also revealed another function of MATH5 in cell survival. Based on the increased apoptotic cells from Math5-lineage in Math5-null embryonic retinas, MATH5 could suppress the apoptosis of retinal cell types during normal embryogenesis. The spatiotemporal pattern of these apoptotic cells suggests their identities as RGCs and amacrine cells because most of these cells are located near the innermost region (the presumptive GCL) of Math5-null retinas and are found soon after the activation of lineage reporter GFP (Figure 6). Future experiments are needed to address how MATH5 regulates the apoptosis. Nevertheless, it is likely that the apoptosis partially accounts for the reduced cell numbers of RGCs or amacrine cells in adult Math5-null retina.

\section{Materials and methods \\ Animals}

The Math5-lacZ and Math5-Cre knock-in mice were generated previously in our laboratory [12,27]. $C M V-\beta$ actin-Cre [28], R26R-lacZ [29], xstpx-lacZ [30], and Z/ $E G$ [31] mice were obtained from The Jackson Laboratory (Bar Harbor, Maine). PCR genotyping of the reporter mice was performed according to protocols provided by The Jackson Laboratory. Embryos were identified as E0.5 at noon on the day at which vaginal plugs were first observed. All animal experiments performed in this study were approved by the University Committee of Animal Resources (UCAR) at University of Rochester.

\section{Immunohistochemistry}

Staged mouse embryos were dissected and fixed in $4 \%$ paraformaldehyde (PFA) in PBS at $4^{\circ} \mathrm{C}$ for $1-2$ hours. Isolated postnatal and adult retinas were fixed for 30 minutes. Fixed samples were saturated in $20 \%$ sucrose in PBS and embedded in Tissue-Tek O.C.T. (Sakura, Torrance, CA) for cryosections cut at a thickness of 14 $\mu \mathrm{m}$. Immunofluorescent labeling and BrdU pulse-labeling experiments were performed as described [12]. Working dilutions and sources of antibodies used in this study are listed in Table 1. Alexa-conjugated secondary antibodies were obtained from Molecular Probes (Eugene, OR) and were used at a concentration of 1:1,000. Images were digitally captured using a Zeiss 510 META confocal microscope. To quantify immunolabeled retinal cells, the number of cells per retinal section or within $350 \mu \mathrm{m}$ field length was counted for each retina and at least five retinas were averaged for each cell type. All results were analyzed for student's t-test significance using Microsoft Excel program.

\section{Additional material}

\begin{abstract}
Additional file 1: Supplemental Figure S1. Changes of retinal cell subtypes in Math5-null retinas. Retinal sections from the control (top panels) and Math5-null (bottom panels) mice at P28 were

immunolabeled with cell type-specific markers (green) and nuclear counterstained with propidium iodide (PI, red). PAX6+ amacrine cells in the INL (A), CHX10+ bipolar cells (B), Go $\alpha+$ ON-bipolar cells (C), PKC $\alpha+$ rod bipolar cells (D), recoverin+ Type 2 OFF-cone bipolar cells (E) and $\mathrm{NRL}+$ rod photoreceptors $(G)$ are reduced in number in Math5-null retina. No overt change is seen in calbindin+ horizontal cells (F). Scale bar equals $100 \mu \mathrm{m}$.

Additional file 2: Supplemental Figure S2. Lineage analysis of Math5-expressing cells. (A) Schematic description of the Cre/loxP mediated conditional activation of reporter genes using Math5-Cre and lacZ or EGFP reporter mouse lines. (B-D) Expression comparison of three reporter genes in retinas. Adult retinal sections from three different reporter lines, CMV-Cre/t; R26R-lacZ, CMV-Cre/t; xstpx-lacZ and CMV-Crel +; Z/EG were immunolabeled with anti-lacZ or anti-GFP (green). In contrast to the biased expression of lac $Z$ expression in the cells of the $\mathrm{GCL}$ and the INL in $R 26 R$ and $x \operatorname{stp} x-l a c Z$ mice ( $B$ and $C$ ), the GFP expression in the CMV-Cre/t; Z/EG retina reveals a uniform distribution of GFP+ cells in all retinal cells (D). Scale bar equals $100 \mu \mathrm{m}$.
\end{abstract}

Additional file 3: Supplemental Figure S3. Line Analysis of retinal cell types from Math5-lineage. Retinal sections from the indicated developmental stages were immunolabeled with cell type/proliferation markers (red) and anti-GFP (green). There was no discernible change in the number of PAX6+/GFP+ amacrine cells in the INL within adult Math5-null retina (B), while there was an increase in NRL+/GFP+ rods, ISL1+/GFP+ cells, PROX1+/GFP+ amacrine cells, and PROX1+/GFP+ displaced amacrine cells (C and D). No overt change was detected in calbindin+/GFP+ and p27kip1+ cells (E and F). Scale bar is $100 \mu \mathrm{m}$.

Additional file 4: Supplemental Figure S4. Comparison of cell proliferation rate in the normal and Math5-null retinas. Retinal sections from the indicated developmental stages were immunolabeled with cell proliferation markers $\mathrm{BrdU}$ or $\mathrm{PH} 3$ (red) and anti-GFP (green). The number of GFP+/BrdU+ and GFP+/PH3+ proliferating cells in Math5null retinas is comparable to that in the controls at E12.5 and E17.5. Scale bar equals $100 \mu \mathrm{m}$.

Additional file 5: Supplemental Figure S5. Altered expression of RGCs factors and non-RGCs factors in the developing Math5-lineage cells in the absence of Math5. Retinal sections from the indicated developmental stages were immunolabeled with cell type markers (red) and anti-GFP (green). At E12.5, GFP+/BHLHB5+ or GFP+/NEUROD1+ cells are rarely detected in the control retina, whereas their cohorts are seen in the Math5-null retina (A and B). At E17.5, more GFP+/BHLBHB5+ cohorts are seen in the GCL as well as GFP+/NEUROD1+ cohorts in the outermost NBL in the Math5-null retina (C and $\mathrm{G}$ ). While fewer GFP $+/ \mathrm{BRN} 3 \mathrm{~B}+, \mathrm{GFP}+/ \mathrm{SL} 1+$, and GFP+/calretinin + cohorts are detected in the Math5-null retina (D-F), the GFP+/RXR + cells are increased in number in the outermost NBL $(H)$. Enlarged views of boxed regions in $A$ and $B$ are shown, respectively. Scale bars equal $100 \mu \mathrm{m}$ in A (applies to A and B), C (applies to $\mathrm{C}-\mathrm{H}$ ).

\section{Acknowledgements}

We thank the members of the Gan Laboratory for helpful discussions and technical assistance. We thank Dr. R. L. Chow for providing the anti-Vsx1 antibody. This work was supported by NIH grants EY013426 to L.G., Research to Prevent Blindness Career Development Award to R.T.L., and the RPB grant to Department of Ophthalmology, University of Rochester Medical Center. 


\section{Author details}

${ }^{1}$ Flaum Eye Institute, University of Rochester School of Medicine and Dentistry, Rochester, NY 14642, USA. ${ }^{2}$ Department of Ophthalmology, University of Rochester School of Medicine and Dentistry, Rochester, NY 14642, USA. ${ }^{3}$ Department of Neurobiology and Anatomy, University of Rochester School of Medicine and Dentistry, Rochester, NY 14642, USA.

\section{Authors' contributions}

LF writes the manuscript and is responsible for all figures except Figure 5. ZX and QD are responsible for Figure 5. XX and LG are responsible for generating Math5-HA mice. LG is responsible for experimental design. LG and RL revise the manuscript. All authors read and approved the final manuscript.

\section{Competing interests}

The authors declare that they have no competing interests.

Received: 29 October 2010 Accepted: 18 November 2010 Published: 18 November 2010

\section{References}

1. Cepko CL, Austin CP, Yang X, Alexiades M, Ezzeddine D: Cell fate determination in the vertebrate retina. PNAS 1996, 93:589-595.

2. Livesey FJ, Cepko CL: VERTEBRATE NEURAL CELL-FATE DETERMINATION: LESSONS FROM THE RETINA. Nat Rev Neurosci 2001, 2:109-118.

3. Young RW: Cell differentiation in the retina of the mouse. The Anatomical Record 1985, 212:199-205.

4. Hatakeyama J, Tomita K, Inoue T, Kageyama R: Roles of homeobox and bHLH genes in specification of a retinal cell type. Development 2001, 128:1313-1322.

5. Inoue T, Hojo M, Bessho Y, Tano Y, Lee JE, Kageyama R: Math3 and NeuroD regulate amacrine cell fate specification in the retina. Development (Cambridge, England) 2002, 129:831-842.

6. Hatakeyama J, Kageyama R: Retinal cell fate determination and bHLH factors. Seminars in Cell \& Developmental Biology Protein Misfolding and Human Disease and Developmental Biology of the Retina 2004, 15:83-89.

7. Wang JC-C, Harris WA: The role of combinational coding by homeodomain and bHLH transcription factors in retinal cell fate specification. Developmental Biology 2005, 285:101-115.

8. Hatakeyama J, Tomita K, Inoue T, Kageyama R: Roles of homeobox and bHLH genes in specification of a retinal cell type. Development 2001, 128:1313-1322.

9. Wang SW, Kim BS, Ding K, Wang H, Sun D, Johnson RL, Klein WH, Gan L: Requirement for math5 in the development of retinal ganglion cells. Genes \& Development 2001, 15:24-29.

10. Brown NL, Patel S, Brzezinski J, Glaser T: Math5 is required for retinal ganglion cell and optic nerve formation. Development 2001, 128:2497-2508.

11. Kay JN, Finger-Baier KC, Roeser T, Staub W, Baier H: Retinal Ganglion Cell Genesis Requires lakritz, a Zebrafish atonal Homolog. Neuron 2001, 30:725-736.

12. Yang Z, Ding K, Pan L, Deng M, Gan L: Math5 determines the competence state of retinal ganglion cell progenitors. Dev Biol 2003, 264:240-254

13. Kanadia RN, Cepko CL: Alternative splicing produces high levels of noncoding isoforms of bHLH transcription factors during development. Genes Dev 2010, 24:229-234.

14. Morrow EM, Furukawa T, Lee JE, Cepko CL: NeuroD regulates multiple functions in the developing neural retina in rodent. Development 1999, 126:23-36.

15. Pennesi ME, Cho J-H, Yang Z, Wu SH, Zhang J, Wu SM, Tsai M-J: BETA2/ NeuroD1 Null Mice: A New Model for Transcription Factor-Dependent Photoreceptor Degeneration. I Neurosci 2003, 23:453-461.

16. Feng L, Xie X, Joshi PS, Yang Z, Shibasaki K, Chow RL, Gan L: Requirement for Bhlhb5 in the specification of amacrine and cone bipolar subtypes in mouse retina. Development 2006, 133:4815-4825.

17. Turner $\mathrm{DL}$, Cepko $\mathrm{CL}: \mathrm{A}$ common progenitor for neurons and glia persists in rat retina late in development. Nature 1987, 328:131-136.

18. Holt CE, Bertsch TW, Ellis HM, Harris WA: Cellular determination in the Xenopus retina is independent of lineage and birth date. Neuron 1988, 1:15-26.
19. Wetts R, Fraser SE: Multipotent precursors can give rise to all major cell types of the frog retina. Science 1988, 239:1142-1145.

20. Turner DL, Snyder EY, Cepko CL: Lineage-independent determination of cell type in the embryonic mouse retina. Neuron 1990, 4:833-845.

21. Vetter ML, Brown NL: The role of basic helix-loop-helix genes in vertebrate retinogenesis. Semin Cell Dev Biol 2001, 12:491-498.

22. Furukawa T, Morrow EM, Cepko CL: Crx, a novel otx-like homeobox gene, shows photoreceptor-specific expression and regulates photoreceptor differentiation. Cell 1997, 91:531-541.

23. Ohsawa R, Kageyama R: Regulation of retinal cell fate specification by multiple transcription factors. Brain Res 2008, 1192:90-98.

24. Le TT, Wroblewski E, Patel S, Riesenberg AN, Brown NL: Math5 is required for both early retinal neuron differentiation and cell cycle progression. Dev Biol 2006, 295:764-778

25. Mu X, Fu X, Sun H, Liang S, Maeda H, Frishman LJ, Klein WH: Ganglion Cells Are Required for Normal Progenitor-Cell Proliferation but Not CellFate Determination or Patterning in the Developing Mouse Retina. Current Biology 2005, 15:525-530.

26. Brown NL, Kanekar S, Vetter ML, Tucker PK, Gemza DL, Glaser T: Math5 encodes a murine basic helix-loop-helix transcription factor expressed during early stages of retinal neurogenesis. Development 1998 , 125:4821-4833.

27. Wang SW, Kim BS, Ding K, Wang H, Sun D, Johnson RL, Klein WH, Gan L: Requirement for math5 in the development of retinal ganglion cells. Genes Dev 2001, 15:24-29.

28. Schwenk FB, Udo, Rajewsky, Klaus: A cre-transgenic mouse strain for the ubiquitous deletion of loxP-flanked gene segments including deletion in germ cells. Nucleic Acids Research 1995, 23:5080-5081.

29. Soriano P: Generalized lacZ expression with the ROSA26 Cre reporter strain. Nat Genet 1999, 21:70-71.

30. Zinyk DL, Mercer EH, Harris E, Anderson DJ, Joyner AL: Fate mapping of the mouse midbrain-hindbrain constriction using a site-specific recombination system. Current Biology 1998, 8:665-672.

31. Novak A, Guo C, Yang W, Nagy A, Lobe CG: Z/EG, a double reporter mouse line that expresses enhanced green fluorescent protein upon Cre-mediated excision. genesis 2000, 28:147-155.

32. Xiang M, Zhou L, Macke JP, Yoshioka T, Hendry SH, Eddy RL, Shows TB, Nathans J: The Brn-3 family of POU-domain factors: primary structure, binding specificity, and expression in subsets of retinal ganglion cells and somatosensory neurons. J Neurosci 1995, 15:4762-4785.

33. Haverkamp S, Wässle H: Immunocytochemical analysis of the mouse retina. The Journal of Comparative Neurology 2000, 424:1-23.

34. Gabriel R, Witkovsky P: Cholinergic, but not the rod pathway-related glycinergic (All), amacrine cells contain calretinin in the rat retina. Neuroscience Letters 1998, 247:179-182.

35. Araki CM, Hamassaki-Britto DE: Calretinin co-localizes with the NMDA receptor subunit NR1 in cholinergic amacrine cells of the rat retina. Brain Research 2000, 869:220-224.

36. Ding $Q$, Chen H, Xie X, Libby RT, Tian N, Gan L: BARHL2 differentially regulates the development of retinal amacrine and ganglion neurons. $J$ Neurosci 2009, 29:3992-4003.

37. Liu ISC, Chen J-d, Ploder L, Vidgen D, van der Kooy D, Kalnins VI, Mclnnes RR: Developmental expression of a novel murine homeobox gene (Chx10): Evidence for roles in determination of the neuroretina and inner nuclear layer. Neuron 1994, 13:377-393.

38. Dkhissi O, Julien J-F, Wasowicz M, Jeanine Nguyen-Legros ND-T, VersauxBotteri C: Differential expression of GAD65 and GAD67 during the development of the rat retina. Brain Research 2001, 919:242-249.

39. Galli-Resta L, Resta G, Tan S-S, Reese BE: Mosaics of Islet-1-Expressing Amacrine Cells Assembled by Short-Range Cellular Interactions. J Neurosci 1997, 17:7831-7838.

40. Haverkamp S, Haeseleer F, Hendrickson A: A comparison of immunocytochemical markers to identify bipolar cell types in human and monkey retina. Visual Neuroscience 2003, 20:589-600.

41. Elshatory $Y$, Deng $M, X i e X$, Gan L: Expression of the LIM-homeodomain protein Is 1 in the developing and mature mouse retina. J Comp Neurol 2007, 503:182-197.

42. Kubbutat MH, Key G, Duchrow M, Schluter C, Flad HD, Gerdes J: Epitope analysis of antibodies recognising the cell proliferation associated nuclear antigen previously defined by the antibody Ki-67 (Ki-67 protein). J Clin Pathol 1994, 47:524-528. 
43. Swain PK, Hicks D, Mears AJ, Apel IJ, Smith JE, John SK, Hendrickson A, Milam AH, Swaroop A: Multiple Phosphorylated Isoforms of NRL Are Expressed in Rod Photoreceptors. J Biol Chem 2001, 276:36824-36830.

44. Dyer MA, Cepko CL: Control of Müller glial cell proliferation and activation following retinal injury 2000, 3:873-880.

45. Marquardt T, Ashery-Padan R, Andrejewski N, Scardigli R, Guillemot F, Gruss P: Pax6 Is Required for the Multipotent State of Retinal Progenitor Cells. Cell 2001, 105:43-55.

46. de Melo J, Qiu X, Du G, Cristante L, Eisenstat DD: Dlx1, Dlx2, Pax6, Brn3b, and $\mathrm{Ch} \times 10$ homeobox gene expression defines the retinal ganglion and inner nuclear layers of the developing and adult mouse retina. The Journal of Comparative Neurology 2003, 461:187-204.

47. Elshatory Y, Everhart D, Deng M, Xie X, Barlow RB, Gan L: Islet-1 controls the differentiation of retinal bipolar and cholinergic amacrine cells. J Neurosci 2007, 27:12707-12720.

48. Dyer MA, Livesey FJ, Cepko CL, Oliver G: Prox1 function controls progenitor cell proliferation and horizontal cell genesis in the mammalian retina 2003, 34:53-58.

49. Haverkamp S, Ghosh KK, Hirano AA, Wässle H: Immunocytochemical description of five bipolar cell types of the mouse retina. The Journal of Comparative Neurology 2003, 455:463-476.

50. Zhao X, Huang J, Khani SC, Palczewski K: Molecular Forms of Human Rhodopsin Kinase (GRK1). J Biol Chem 1998, 273:5124-5131.

51. Roberts MR, Hendrickson A, McGuire CR, Reh TA: Retinoid X receptor (gamma) is necessary to establish the S-opsin gradient in cone photoreceptors of the developing mouse retina. Investigative Ophthalmology \& Visual Science 2005, 46:2897-2904.

52. Chow RL, Snow B, Novak J, Looser J, Freund C, Vidgen D, Ploder L, McInnes RR: Vsx 1, a rapidly evolving paired-like homeobox gene expressed in cone bipolar cells. Mechanisms of Development 2001, 109:315-322.

53. Chow RL, Volgyi B, Szilard RK, Ng D, McKerlie C, Bloomfield SA, Birch DG, McInnes RR: Control of late off-center cone bipolar cell differentiation and visual signaling by the homeobox gene Vsx1. PNAS 2004, 101:1754-1759.

\section{Submit your next manuscript to BioMed Central and take full advantage of:}

- Convenient online submission

- Thorough peer review

- No space constraints or color figure charges

- Immediate publication on acceptance

- Inclusion in PubMed, CAS, Scopus and Google Scholar

- Research which is freely available for redistribution

Submit your manuscript at www.biomedcentral.com/submit
Biomed Central 